COLO-HEP-310

\title{
Smooth Random Surfaces from Tight Immersions?
}

\author{
C.F. Baillie \\ Physics Dept. \\ University of Colorado \\ Boulder, CO 80309 \\ USA \\ D.A. Johnston \\ Dept. of Mathematics \\ Heriot-Watt University \\ Riccarton \\ Edinburgh, EH14 4AS \\ Scotland
}

October 24, 2018

\begin{abstract}
We investigate actions for dynamically triangulated random surfaces that consist of a gaussian or area term plus the modulus of the gaussian curvature and compare their behavior with both gaussian plus extrinsic curvature and "Steiner" actions.
\end{abstract}


Considerable effort has recently been devoted to exploring modifications of the discretized Polyakov partition function [1] for a random surface

$$
Z=\sum_{T} \int \prod_{i=1}^{N-1} d X_{i}^{\mu} \exp \left(-S_{g}\right),
$$

where the sum over triangulations $\sum_{T}$ means that we have, in effect, a fluid surface. $S_{g}$ is just a simple gaussian action

$$
S_{g}=\frac{1}{2} \sum_{<i j>}\left(X_{i}^{\mu}-X_{j}^{\mu}\right)^{2},
$$

where the $X$ 's live at the vertices of the triangulation and the sum $\langle i j\rangle$ is over all the edges. Earlier work [2] had made it clear that this action (and variations, such as area and edge length actions [3, 4]) failed to lead to a sensible continuum theory because the string tension did not scale so, inspired by analytical work on QCD strings and biological membranes [5], an extrinsic curvature or "stiffness" term was added

$$
S_{e}=\sum_{\Delta_{i}, \Delta_{j}}\left(1-n_{i} \cdot n_{j}\right)
$$

where $n_{i}, n_{j}$ are the normals on neighboring triangles $\Delta_{i}, \Delta_{j}$. Simulations of $S_{g}+\lambda S_{e}$, called the gaussian plus extrinsic curvature (GPEC) action, seemed to indicate that there was a second order phase transition (the "crumpling transition") from a small $\lambda$ crumpled phase to a large $\lambda$ smooth phase at which one might hope to define a non-trivial continuum theory [6]. More recent simulations of larger surfaces suggest, however, that the transition is not second order 17, 81 1 but that the string tension may possibly still be scaling correctly.

Simpler spin systems on dynamical triangulations, such as Ising and Potts models [10], provide some reassurance that a non-trivial theory may be lurking at the crumpling transition because they display third order transitions and still have a sensible continuum limit. Nonetheless, the rather murky behavior seen in 7 , 8 prompts the question of whether actions with a sharper phase transition can be found. One geometrically appealing suggestion was the Steiner action put forward in [11], and simulated microcanonically in 12

$$
S_{\text {steiner }}=\frac{1}{2} \sum_{<i j>}\left|X_{i}^{\mu}-X_{j}^{\mu}\right| \theta\left(\alpha_{i j}\right),
$$

where $\theta\left(\alpha_{i j}\right)=\left|\pi-\alpha_{i j}\right|$ and $\alpha_{i j}$ is the angle between the embedded neighboring triangles with common link $\langle i j\rangle$. It was pointed out in [13] that the grand canonical partition function diverged for $S_{\text {steiner }}$ alone, so we conducted some exploratory simulations of various actions combining edge-length, area or gaussian terms with $S_{\text {steiner }}$ finding particularly sharp transitions for Area $+\lambda S_{\text {steiner }}$ and $S_{g}+\lambda S_{\text {steiner }}$ actions [14]. As this initial work is on the same small triangulations that indicated a second order transition for the GPEC action we cannot claim this is strong evidence for a sharp transition with the Steiner actions, without further results from larger lattices [15].

In this paper we will explore a further possibility for a random surface action which, like the Steiner term, is a natural object to consider from a geometrical point of view. For a curve $C$ embedded in three dimensions it was shown by Fenchel that

$$
\frac{1}{\pi} \int_{C}|\kappa| d s \geq 2
$$

where $\kappa$ is the curvature and the equality holds when $C$ is a plane convex curve [16]. For a surface $M$ imbedded in three dimensions the Gauss-Bonnet theorem tells us that

$$
\frac{1}{2 \pi} \int_{M} K d S=\chi(M)
$$

where $K$ is now the Gaussian curvature of the surface and $\chi$ is the Euler characteristic. As this is a topological invariant it tells us nothing about the configuration of the surface. To get the equivalent of

\footnotetext{
${ }^{1}$ Unlike the GPEC action on rigid lattices, which almost certainly is [9].
} 
Fenchel's equ.(5) we take a modulus sign in equ.(6) and find 7

$$
\frac{1}{2 \pi} \int_{M}|K| d S \geq 4-\chi(M)
$$

with the equality holding when the surface is imbedded as a convex surface in three dimensional space. This term discretizes to

$$
S_{\text {tight }}=\sum_{i}\left|2 \pi-\sum_{j(i)} \phi_{i j}\right|
$$

where the outer sum is over all the vertices of the triangulation and the inner sum is round the neighbors $j$ of a node $i . \phi_{i j}$ is the angle subtended by the $j$ th triangle at the $i$ th vertex - see Fig.1. The behavior of $S_{\text {tight }}$ was measured for the GPEC action in [8] (rather than being included in the action), where it was found to correlate closely with the extrinsic curvature - dropping sharply in value at the crumpling transition. This raises the hope that including $S_{\text {tight }}$ in the dynamics may be sufficient to give a crumpling transition without the assistance of an extrinsic curvature term.

We will examine the phase structure of both $S_{1}=S_{g}+\lambda S_{\text {tight }}$

$$
S_{1}=\frac{1}{2} \sum_{<i j>}\left(X_{i}^{\mu}-X_{j}^{\mu}\right)^{2}+\lambda \sum_{i}\left|2 \pi-\sum_{j(i)} \phi_{i j}\right|
$$

and $S_{2}=$ Area $+\lambda S_{\text {tight }}$

$$
S_{2}=\sum_{\Delta} A_{\Delta}+\lambda \sum_{i}\left|2 \pi-\sum_{j(i)} \phi_{i j}\right|
$$

where $A_{\Delta}$ is the area of a triangle $\Delta$, in what follows. To do this we employ our by now standard set of observables. We included a local factor in the measure for compatibility with our earlier simulations which can be exponentiated to give

$$
S_{m}=\frac{d}{2} \sum_{i} \log \left(q_{i}\right)
$$

where $q_{i}$ is the number of neighbors of point $i$, and $d=3$ dimensions. We thus simulated $S_{1,2}+S_{m}$. We measured $\left\langle S_{m}>\right.$ and the mean maximum number of neighbors $\left\langle\max \left(q_{i}\right)>\right.$ to get some idea of the behavior of the intrinsic geometry. The extrinsic geometry was observed by measuring $\left\langle S_{\text {tight }}\right\rangle$ and its associated specific heat

$$
C=\frac{\lambda^{2}}{N}\left(<S_{\text {tight }}^{2}>-<S_{\text {tight }}>^{2}\right)
$$

as well as the gyration radius $X 2$, a measure of the mean size of the surface as seen in the embedding space,

$$
X 2=\frac{1}{9 N(N-1)} \sum_{i j}\left(X_{i}^{\mu}-X_{j}^{\mu}\right)^{2} q_{i} q_{j} .
$$

For $S_{1}$ the expectation value of $S_{g}$ can be shown to be $d(N-1) / 2$ by exactly the same argument that is used to give this result for the GPEC action, because $S_{\text {tight }}$ shares the scale invariance of the extrinsic curvature term in the GPEC action. This serves as a useful check of equilibration in this case. A further useful check is provided by removing the modulus sign in equ.(8) which should then sum to give $4 \pi$ for every configuration by the Gauss-Bonnet theorem.

The simulation used a Monte Carlo procedure which we have described in some detail elsewhere [17. It first goes through the mesh moving the $X$ 's, carrying out a Metropolis accept/reject at each step, and then goes through the mesh again carrying out the "flip" moves on the links, again applying a Metropolis accept/reject at each stage. The entire procedure constitutes a sweep. Due to the correlated nature of the data, a measurement was taken every tenth sweep and binning techniques were used to analyze the errors. We carried out $10 \mathrm{~K}$ thermalization sweeps followed by $30 \mathrm{~K}$ measurement sweeps for each data point. The acceptance for the $X$ move was monitored and the size of the shift was adjusted to maintain an acceptance of around 50 percent. The acceptance for the flip move was also measured, but in this

${ }^{2}$ As a particular case of the theorem: Let $M^{n}$ be a compact oriented $C^{\infty}$ manifold immersed in $E^{n+N}$, such that the total absolute curvature equals 2 . Then $M^{n}$ belongs to a linear subvariety of dimension $n+1$, and is imbedded as a convex hypersurface in $E^{n+1}$. The converse is also true 16 . 
case there is nothing to adjust, so as for GPEC actions this dropped with increasing $\lambda$ (but was still appreciable even for quite large $\lambda$ ).

If we look at the numerical results for $S_{1}\left(S_{g}+\lambda S_{\text {tight }}\right)$ in Table 1 first

\begin{tabular}{|c|c|c|c|c|c|c|c|}
\hline$\lambda$ & sweeps & $S_{g}$ & $S_{m}$ & $S_{\text {tight }}$ & $C_{\text {tight }}$ & $X 2$ & $\max \left(q_{i}\right)$ \\
\hline 0.500 & $30 \mathrm{~K}$ & $106.62(0.03)$ & $122.79(0.00)$ & $109.94(0.03)$ & $0.46(0.00)$ & $2.40(0.01)$ & $12.07(0.00)$ \\
1.000 & $30 \mathrm{~K}$ & $106.33(0.01)$ & $123.60(0.00)$ & $66.40(0.00)$ & $0.77(0.00)$ & $2.54(0.01)$ & $10.92(0.00)$ \\
1.250 & $30 \mathrm{~K}$ & $106.34(0.05)$ & $123.76(0.00)$ & $54.96(0.01)$ & $0.84(0.00)$ & $2.53(0.01)$ & $10.70(0.00)$ \\
1.500 & $30 \mathrm{~K}$ & $106.45(0.03)$ & $123.83(0.00)$ & $46.70(0.02)$ & $0.86(0.00)$ & $2.80(0.04)$ & $10.59(0.00)$ \\
1.750 & $30 \mathrm{~K}$ & $106.58(0.10)$ & $123.89(0.00)$ & $40.77(0.02)$ & $0.91(0.00)$ & $2.77(0.05)$ & $10.52(0.00)$ \\
2.000 & $30 \mathrm{~K}$ & $107.25(0.21)$ & $123.91(0.00)$ & $36.10(0.05)$ & $0.93(0.00)$ & $2.94(0.12)$ & $10.49(0.00)$ \\
2.250 & $30 \mathrm{~K}$ & $106.28(0.02)$ & $123.92(0.00)$ & $32.11(0.01)$ & $0.90(0.00)$ & $2.16(0.00)$ & $10.48(0.00)$ \\
2.500 & $30 \mathrm{~K}$ & $106.34(0.07)$ & $123.91(0.00)$ & $29.42(0.02)$ & $0.90(0.00)$ & $2.17(0.01)$ & $10.48(0.00)$ \\
3.000 & $30 \mathrm{~K}$ & $106.52(0.08)$ & $123.90(0.00)$ & $25.18(0.00)$ & $0.82(0.00)$ & $2.14(0.01)$ & $10.49(0.00)$ \\
3.500 & $30 \mathrm{~K}$ & $106.48(0.10)$ & $123.88(0.00)$ & $22.53(0.01)$ & $0.78(0.00)$ & $2.15(0.01)$ & $10.51(0.00)$ \\
4.000 & $30 \mathrm{~K}$ & $106.02(0.05)$ & $123.84(0.00)$ & $20.56(0.01)$ & $0.73(0.00)$ & $2.12(0.02)$ & $10.53(0.00)$ \\
4.500 & $30 \mathrm{~K}$ & $106.08(0.37)$ & $123.80(0.00)$ & $19.15(0.00)$ & $0.68(0.00)$ & $2.20(0.07)$ & $10.57(0.01)$ \\
\hline
\end{tabular}

Table 1

Results for $S_{1}, N=72$

we can see that $S_{\text {tight }}$ does indeed drop off with increasing $\lambda$ just like the extrinsic curvature. The behavior of $S_{m}$ and $\max \left(q_{i}\right)$ is also reminiscent of the GPEC action, with the internal geometry becoming more regular with increasing $\lambda$. However, the specific heat $C$ shows only a modest cusp at around $\lambda=2.00$ as can be seen in Fig.2, which should be contrasted with the larger peaks seen on these small meshes for both the GPEC and Steiner actions. Similarly X2, plotted in Fig.3, shows no sign of a crumpling transition, with only a small increase in the region of the cusp in $C$, before it rapidly drops off. The value of the gaussian term is close to the expected $d(N-1) / 2$, assuring us that the results are equilibrated and both the metropolis and flip acceptances are reasonable for all the values of $\lambda$ simulated, so we can be sure that the simulation is performing as it should. We also measured the value of the gaussian curvature using $S_{\text {tight }}$ with the modulus sign removed and found, as expected (our surfaces have spherical topology), $4 \pi$ for every surface generated. Visual inspection of "snapshots" of the surfaces that arise in the simulation confirms the absence of a phase transition, with surfaces looking similar for all of the $\lambda$ values simulated. One of these for $\lambda=4.0$, but which is typical of all the others, is shown in Fig.4 and is obviously not smooth. Even at the largest $\lambda$ values simulated the surfaces are still some way from satisfying the lower bound of $4 \pi$ on $S_{\text {tight }}$, so it would appear that the disordering effect of $S_{g}$ overcomes $S_{\text {tight }}$ for all $\lambda$.

The behavior of $S_{2}\left(\right.$ Area $\left.+\lambda S_{\text {tight }}\right)$ is rather bizarre, as can be seen in Table 2 .

\begin{tabular}{|c|c|c|c|c|c|c|c|}
\hline$\lambda$ & sweeps & Area & $S_{m}$ & $S_{\text {tight }}$ & $C_{\text {tight }}$ & $X 2$ & $\max \left(q_{i}\right)$ \\
\hline 0.500 & $30 \mathrm{~K}$ & $106.05(0.03)$ & $122.26(0.00)$ & $119.51(0.09)$ & $0.70(0.01)$ & $10.67(0.17)$ & $12.66(0.00)$ \\
1.000 & $30 \mathrm{~K}$ & $105.81(0.13)$ & $122.88(0.00)$ & $61.28(0.43)$ & $0.86(0.01)$ & $22.60(3.46)$ & $11.91(0.01)$ \\
1.500 & $30 \mathrm{~K}$ & $105.78(0.18)$ & $122.95(0.01)$ & $38.55(0.28)$ & $0.63(0.01)$ & $113.70(11.84)$ & $11.80(0.02)$ \\
1.750 & $30 \mathrm{~K}$ & $106.11(0.38)$ & $122.93(0.02)$ & $34.01(0.50)$ & $0.69(0.01)$ & $141.42(42.03)$ & $11.89(0.05)$ \\
2.000 & $30 \mathrm{~K}$ & $105.50(0.10)$ & $122.73(0.03)$ & $31.63(0.14)$ & $0.79(0.03)$ & $33.69(10.03)$ & $12.42(0.07)$ \\
2.250 & $30 \mathrm{~K}$ & $104.85(0.08)$ & $122.70(0.01)$ & $29.55(0.06)$ & $0.87(0.03)$ & $25.39(3.94)$ & $12.57(0.03)$ \\
3.000 & $30 \mathrm{~K}$ & $106.04(0.06)$ & $122.34(0.00)$ & $22.21(0.02)$ & $0.61(0.00)$ & $10.31(0.10)$ & $13.60(0.00)$ \\
3.500 & $30 \mathrm{~K}$ & $105.59(0.09)$ & $122.19(0.00)$ & $20.10(0.01)$ & $0.56(0.00)$ & $10.28(0.03)$ & $14.08(0.01)$ \\
4.000 & $30 \mathrm{~K}$ & $106.68(0.25)$ & $122.12(0.01)$ & $18.80(0.01)$ & $0.54(0.00)$ & $10.50(0.15)$ & $14.16(0.02)$ \\
4.500 & $30 \mathrm{~K}$ & $106.26(0.23)$ & $121.98(0.01)$ & $17.68(0.01)$ & $0.50(0.00)$ & $11.17(0.18)$ & $14.67(0.02)$ \\
\hline
\end{tabular}

Table 2

Results for $S_{2}, N=72$

Again $S_{\text {tight }}$ decreases with increasing $\lambda$ but there is no obvious peak in the specific heat. $\max \left(q_{i}\right)$ now increases at large $\lambda$ and there is a huge peak in $X 2$ at around $\lambda=2.5$. For $\lambda>2.5$ the surfaces generated look rather similar to those produced by $S_{1}$, but near $\lambda=2.5$ they are very long, jointed linear structures 
such as that in Fig.5. On its own the area action gives surfaces that are collections of long thin spikes emanating from a central point, and this is also seen for $S_{2}$ at small $\lambda$. It appears that adding $S_{\text {tight }}$ gives (approximately) very long, thin ellipsoids which, while satisfying the convexity property, are not the generic smooth surfaces that we envisaged. From the evidence of the specific heat there is little sign of a phase transition in this region that might be used to define a continuum limit, though it is always possible that a higher order transition may be present.

Our conclusions are rather disappointing from the point of view of finding candidate random surface actions which might be used similarly to the GPEC action to hunt for a non-trivial continuum string theory. $S_{1}$ shows little sign of a transition at all, apart from a modest bump in the specific heat, and $S_{2}$, whilst adding to the bestiary of amusing pathologies that have been observed with various random surface models, also looks unpromising. It may of course be possible to incorporate $S_{\text {tight }}$ as an additional term in the GPEC action in order to tune the couplings to see if the approach to the continuum limit could be optimized. A further possibility that might be worth pursuing is looking at the effect of self-avoidance, which completely changes the behavior of GPEC actions [18].

This work was supported in part by NATO collaborative research grant CRG910091. CFB is supported by DOE under contract DE-FG02-91ER40672 and by NSF Grand Challenge Applications Group Grant ASC-9217394. The computations were performed on workstations at Heriot-Watt University. We would like to thank R.D. Williams for help in developing initial versions of the dynamical mesh code. 


\section{References}

[1] A. M. Polyakov, Phys. Lett. B103 (1981) 207.

[2] J. Ambjorn, B. Durhuus and J. Frohlich, Nucl. Phys. B257 (1985) 433;

D. Boulatov, V. Kazakov, I Kostov and A. Migdal, Nucl. Phys. B275 (1986) 641;

A. Billoire and F. David, Nucl. Phys. B275 (1986) 617;

J. Jurkiewicz, A. Krzywicki and B. Petersson, Phys. Lett. B168 (1986) 273.

[3] J. Ambjorn, B. Durhuus and J. Frohlich, Nucl. Phys. B257 (1985) 433.

[4] J. Ambjorn and B. Durhuus, Phys. Lett. B118 (1987) 253.

[5] A. Polyakov, Nucl. Phys. B268 (1986) 406;

H. Kleinert, Phys. Lett. B174 (1986) 335;

W. Helfrich, J. Phys. 46 (1985) 1263.

[6] S. Catterall, Phys. Lett. B220 (1989) 207;

C. F. Baillie, D. A. Johnston and R. D. Williams, Nucl. Phys. B335 (1990) 469;

R. Renken and J. Kogut, Nucl. Phys. B354 (1991) 328;

S. Catterall, Phys. Lett. B243 (1990) 121;

C. F. Baillie, R. D. Williams, S. M. Catterall and D. A. Johnston, Nucl. Phys. B348 (1991) 543;

S. Catterall, D. Eisenstein, J. Kogut and R. Renken, Nucl. Phys. B366 (1991) 647.

[7] J. Ambjorn, J. Jurkiewicz, S. Varsted, A. Irback and B. Petersson, Phys. Lett. B275 (1992) 295; J. Ambjorn, J. Jurkiewicz, S. Varsted, A. Irback and B. Petersson, "The Theory of Dynamical Random Surfaces with Extrinsic Curvature", Niels Bohr preprint NBI-HE-92-40.

[8] M. Bowick, P. Coddington, L. Han, G. Harris and E. Marinari, "The Phase Diagram of Fluid Random Surfaces with Extrinsic Curvature", Syracuse preprint SU-HEP-4241-517.

[9] R. Harnish and J. Wheater, Nucl. Phys. B350 (1991) 861;

J. Wheater and P. Stephenson, "On the Crumpling Transition in Random Surfaces", Oxford preprint OUTP 92/40P (hep-lat 9301007).

[10] C. F. Baillie and D. A. Johnston, Mod. Phys. Lett. A 5 (1990) 1671.

[11] R. V. Ambartzumian, G. K. Savvidy, K. G. Savvidy and G. S. Sukiasian, Phys. Lett. B275 (1992) 99.

[12] C. F. Baillie and D. A. Johnston, Phys. Rev. D45 (1992) 3326.

[13] B. Durhuus and T. Jonsson, Phys. Lett. B297 (1992) 271.

[14] C. F. Baillie, D. Espriu and D. A. Johnston, "Steiner Variations on Random Surfaces", University of Colorado preprint, COLO-HEP-297.

[15] C. F. Baillie and D. A. Johnston, work in progress.

[16] T. Willmore, "Total Curvature in Riemannian Geometry", Wiley 1982.

[17] C. F. Baillie, D. A. Johnston and R. D. Williams, Comput. Phys. Commun. 58 (1990) 105.

[18] C. F. Baillie and D. A. Johnston, Phys. Lett. B273 (1990) 380; ibid B283 (1992) 55; ibid B295 (1992) 249. 


\section{Figure Captions}

Fig. 1. The neighbors and angles used in defining $S_{\text {tight }}$.

Fig. 2. The specific heat $C$ for action $S_{1}$.

Fig. 3. The gyration radius $X 2$ for action $S_{1}$.

Fig. 4. A snapshot of a mesh generated by $S_{1}$ with $\lambda=4.5$.

Fig. 5. A snapshot of a mesh generated by $S_{2}$ with $\lambda=2.5$. 